\title{
Reduced-bias estimator of the Proportional Hazard Premium for heavy-tailed distributions
}

\author{
El hadji Deme ${ }^{(1)}$, Stéphane Girard ${ }^{(2)}$ and Armelle Guillou ${ }^{(3)}$ \\ (1) LERSTAD, Université Gaston Berger de Saint-Louis, Sénégal \\ (2) Team Mistis, INRIA Rhône-Alpes \& Laboratoire Jean Kuntzmann \\ 655, avenue de l'Europe, Montbonnot, 38334 Saint-Ismier Cedex, France \\ (3) Université de Strasbourg et CNRS, IRMA, UMR 7501, \\ 7 rue René Descartes, 67084 Strasbourg cedex, France
}

\begin{abstract}
Many different premium principles have been proposed in the literature. In this paper, we focus on the Proportional Hazard Premium. Its asymptotic normality has been established in the literature under suitable conditions which are not fulfilled in case of heavy-tailed distributions. We thus focus on this framework and propose a reduced-bias approach for the classical estimators. A small simulation study is proposed to illustrate the efficiency of our approach.
\end{abstract}

Keywords: Bias correction · Extreme values · Heavy-tailed distribution · Proportional Hazard Premium · Kernel estimators

\section{Introduction}

Several premium principles have been introduced in the actuarial literature. We refer to Govaerts et al. (1984) for various examples and properties of such principles. One of the most commonly used is the net premium defined for a non-negative loss random variable $X$ with tail distribution function $\bar{F}:=1-F$ as

$$
\pi:=\mathbb{E}(X)=\int_{0}^{\infty} \bar{F}(x) d x .
$$

In general, premiums are required to be greater than or equal to the net premium $\mathbb{E}(X)$ in order to avoid that the insurer loses money on average. One way to achieve this goal consists in introducing an increasing concave function $g$ that maps $[0,1]$ onto $[0,1]$, such that $g(0)=0$ and $g(1)=1$ and to define

$$
\pi(g, F):=\int_{0}^{\infty} g(\bar{F}(x)) d x
$$


An example of such distortion function $g$ is $g(x)=x^{\frac{1}{\rho}}$, for $\rho \geq 1$. It leads to the well-known Proportional Hazard Premium (PHP) defined as the distorted expectation of $X$ (see e.g. Wang, 1995, 1996):

$$
\pi(\rho):=\int_{0}^{\infty}(\bar{F}(x))^{\frac{1}{\rho}} d x .
$$

The parameter $\rho \geq 1$ represents the distortion coefficient or the risk aversion index. It controls the amount of risk loading in the premium. When the parameter is at its minimal value, $\rho=1$, then $\pi(\rho)$ is the net premium $\pi$, and thus there is no loading. The risk loading increases when $\rho$ increases. This class of premium has been extensively studied in the literature in particular as it can also be grounded in economics via Yaari's (1987) dual theory of expected utility.

For high layer with retention level $R>0$, the corresponding PHP can be defined as

$$
\pi_{R}(\rho):=\int_{R}^{\infty}(\bar{F}(x))^{\frac{1}{\rho}} d x
$$

In the case of high-excess loss layers $(R \rightarrow \infty)$, Necir and Boukhetala (2004), Vandewalle and Beirlant (2006) and Necir et al. (2007) have introduced and studied different estimators for $\pi_{R}(\rho)$ based on samples of heavy-tailed claim amounts.

However, in the no-retention case $(R=0)$, the technique of estimation of $\pi(\rho)$ is different from the case $R$ large and has been only recently studied in the literature by Centeno and Andrade e Silva (2005) via bootstrap techniques, and Necir and Meraghni (2009) via empirical processes arguments and extreme value theory. This later approach is also the one used in this paper, but unlike Necir and Meraghni (2009), we deal with the problem of bias of the proposed estimators.

Using the quantile function of $F$ defined as $Q(s):=\inf \{x>0: F(x) \geq s\}, s \in(0,1]$, we may rewrite $\pi(\rho)$ as

$$
\pi(\rho)=-\int_{0}^{1} s^{1 / \rho} d(Q(1-s)) .
$$

Let $X_{1}, \ldots, X_{n}$ be a sample of independent and identically distributed random variables from $F$ and let $X_{1, n} \leq \ldots \leq X_{n, n}$ denote the order statistics. Set $F_{n}$ the empirical distribution function and $Q_{n}$ the empirical quantile function defined as $Q_{n}(s)=X_{j, n}$ for all $(j-1) / n<s \leq j / n$ and $j=1, \ldots, n$ with $Q_{n}(0)=X_{1, n}$.

A natural estimator for $\pi(\rho)$ can be obtained by replacing $Q$ by $Q_{n}$ and using an integration by parts:

$$
\widehat{\pi}_{n}(\rho)=\sum_{j=1}^{n}\left(\left(\frac{j}{n}\right)^{1 / \rho}-\left(\frac{j-1}{n}\right)^{1 / \rho}\right) X_{n-j+1, n} .
$$

The main asymptotic properties of $\widehat{\pi}_{n}(\rho)$ have been established by Jones and Zitikis $(2003)$ : $\widehat{\pi}_{n}(\rho)$ is consistent for all $\rho \geq 1$ provided that $\mathbb{E}|X|^{\eta}<\infty$ for $\eta>\rho$. It is asymptotically normal if $\rho \in[1,2)$ and under the same moment condition for $X$ but this time restricted to $\eta>2 \rho /(2-\rho)$. This paper deals with the estimation problem of the PHP within the class of heavy-tailed distri- 
bution, i.e. we assume that

$$
\bar{F}(x)=x^{-1 / \gamma} \ell_{F}(x)
$$

where $\gamma>0$ is the extreme value index and $\ell_{F}$ is a slowly varying function at infinity satisfying $\ell_{F}(\lambda x) / \ell_{F}(x) \rightarrow 1$ as $x \rightarrow \infty$ for all $\lambda>0$. Moreover we focus our paper on the case $\gamma \in\left(\frac{1}{2}, 1\right)$ and $\rho \in\left[1, \frac{1}{\gamma}\right)$ in order to ensure that the PHP is finite and since in that case the results of Jones and Zitikis (2003) cannot be applied, the second moment of $X$ being infinite.

The estimation of $\gamma$ has been extensively studied in the literature and the most famous estimator is the Hill (1975) estimator defined as

$$
\widehat{\gamma}_{n, k}^{H}=\frac{1}{k} \sum_{j=1}^{k} j\left(\log X_{n-j+1, n}-\log X_{n-j, n}\right)
$$

for an intermediate sequence $k$, i.e. a sequence such that $k \rightarrow \infty$ and $k / n \rightarrow 0$ as $n \rightarrow \infty$.

More generally, Csörgö et al. (1985) extended the Hill estimator into a kernel class of estimators

$$
\widehat{\gamma}_{n, k}^{K}=\frac{1}{k} \sum_{j=1}^{k} K\left(\frac{j}{k+1}\right) Z_{j, k},
$$

where $K$ is a kernel integrating to one and $Z_{j, k}=j\left(\log X_{n-j+1, n}-\log X_{n-j, n}\right)$. Note that the Hill estimator corresponds to the particular case where $K=\underline{K}:=\mathbb{1}_{(0,1)}$.

In this spirit, we propose a kernel-type estimator for the PHP. Indeed, recall that from $(1), \pi(\rho)$ can be rewritten as

$$
\begin{aligned}
\pi(\rho) & =\left\{\left(\frac{k}{n}\right)^{\frac{1}{\rho}} Q\left(1-\frac{k}{n}\right)-\int_{0}^{k / n} s^{1 / \rho} d(Q(1-s))\right\}+\left\{\frac{1}{\rho} \int_{k / n}^{1} s^{\frac{1}{\rho}-1} Q(1-s) d s\right\} \\
& =: \pi_{n, k}^{(1)}(\rho)+\pi_{n, k}^{(2)}(\rho) .
\end{aligned}
$$

Thus, $\pi(\rho)$ can be estimated by

$$
\begin{aligned}
\tilde{\pi}_{n, k}^{K}(\rho) & =\frac{(k / n)^{1 / \rho}}{1-\rho \widehat{\gamma}_{n, k}^{K}} X_{n-k, n}+\sum_{j=k+1}^{n}\left(\left(\frac{j}{n}\right)^{1 / \rho}-\left(\frac{j-1}{n}\right)^{1 / \rho}\right) X_{n-j+1, n} \\
=: & \tilde{\pi}_{n, k}^{(K, 1)}(\rho)+\widetilde{\pi}_{n, k}^{(2)}(\rho) .
\end{aligned}
$$

Note that to estimate $\pi_{n, k}^{(2)}(\rho)$ we use the same trick as for $(2)$, whereas for $\pi_{n, k}^{(1)}(\rho)$ we use a

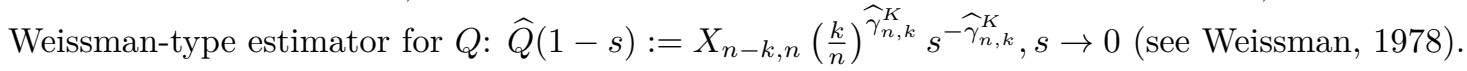

Asymptotic normality for $\tilde{\pi}_{n, k}^{K}(\rho)$ is obviously related to the one of $\widehat{\gamma}_{n, k}^{K}$. As usual in the extreme value framework, to prove such type of results, we need a second-order condition on the function $\mathbb{U}(x)=Q(1-1 / x)$ such as the following: 
Condition $\left(\mathcal{R}_{\mathbb{U}}\right)$. There exist a function $A(x) \rightarrow 0$ as $x \rightarrow \infty$ of constant sign for large values of $x$ and a second order parameter $\omega<0$ such that, for every $x>0$,

$$
\lim _{t \rightarrow \infty} \frac{\log \mathbb{U}(t x)-\log \mathbb{U}(t)-\gamma \log x}{A(t)}=\frac{x^{\omega}-1}{\omega} .
$$

Note that condition $\left(\mathcal{R}_{\mathbb{U}}\right)$ implies that $|A|$ is regularly varying with index $\omega$ (see, e.g. Geluk and de Haan, 1987). It is satisfied for most of the classical distribution functions such as the Pareto, Burr and Fréchet ones.

The paper is organized as follows. In Section 2, we state the main asymptotic properties of the kernel estimator $\widehat{\gamma}_{n, k}^{K}$ from which we deduce the ones of $\widetilde{\pi}_{n, k}^{K}(\rho)$. This result illustrates the fact that this estimator can exhibit severe bias in many situations as in Figure 1 below: The larger $\omega$, the larger the bias. To overcome this problem a reduced-bias approach is also proposed. The efficiency of our method is shown on a small simulation study in Section 3. Section 4 is devoted to the proofs.
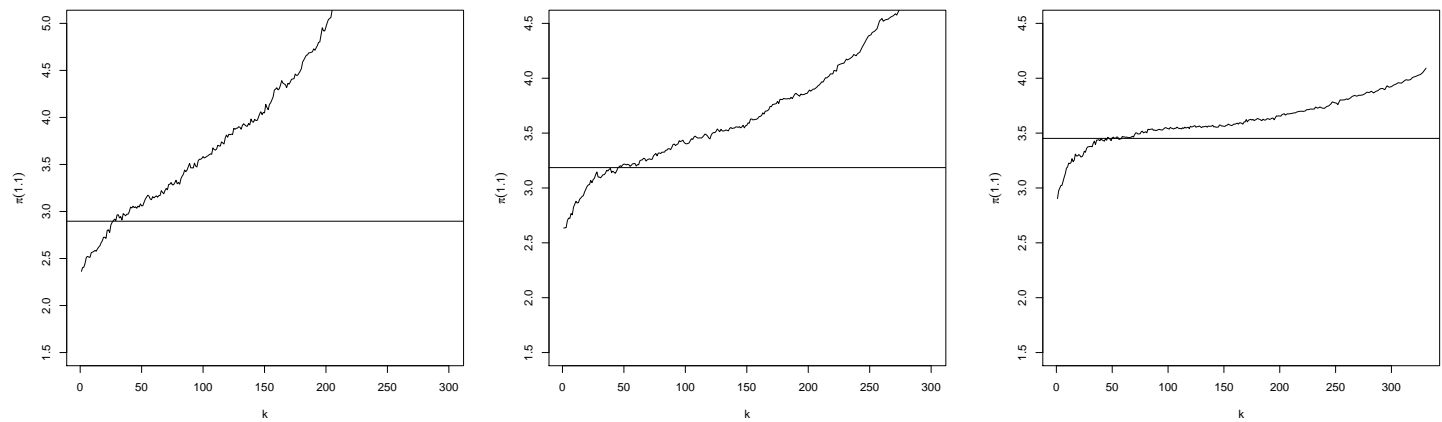

Figure 1: Median of $\widetilde{\pi}_{n, k}^{K}(1.1)$ as a function of $k$ based on 500 samples of size 1000 from a Burr distribution defined as $\bar{F}(x)=\left(1+x^{-\frac{3 \omega}{2}}\right)^{1 / \omega}$. From the left to the right: $\omega=-0.75, \omega=-1$ and $\omega=-1.5$. The horizontal line represents the true value of the premium. 


\section{Main results}

We start to establish some results on the estimator $\widehat{\gamma}_{n, k}^{K}$ in Theorem 1 . Then, the asymptotic normality of the estimator of the PHP $\widetilde{\pi}_{n, k}^{K}(\rho)$ is derived. As it exhibits some bias, we propose a reduced-bias estimator whose asymptotic normality is also obtained.

\subsection{Asymptotic results for the kernel estimator $\widehat{\gamma}_{n, k}^{K}$}

To establish the asymptotic normality of the kernel estimator $\widehat{\gamma}_{n, k}^{K}$, some classical assumptions about the kernel are needed:

Condition $(\mathcal{K})$. Let $K$ be a function defined on $(0,1]$

(i) $K(s) \geq 0$, whenever, $0<s \leq 1$ and $K(1)=0$;

(ii) $K(\cdot)$ is differentiable, nonincreasing and right continuous on $(0,1]$;

(iii) $K$ and $K^{\prime}$ are bounded;

(iv) $\int_{0}^{1} K(u) d u=1$;

(v) $\int_{0}^{1} u^{-1 / 2} K(u) d u<\infty$.

Theorem 1. Let $K$ be a kernel satisfying $(\mathcal{K})$ and assume that the distribution $F$ satisfies $\left(\mathcal{R}_{\mathbb{U}}\right)$. Then, as $k \rightarrow \infty, k / n \rightarrow 0$ and $\sqrt{k} A(n / k)=O(1)$ as $n \rightarrow \infty$, we have

$$
\sqrt{k}\left(\widehat{\gamma}_{n, k}^{K}-\gamma-A(n / k) \int_{0}^{1} s^{-\omega} K(s) d s\right) \stackrel{\mathcal{D}}{=} \gamma \sqrt{\frac{n}{k}} \int_{0}^{1} s^{-1} \mathbb{B}_{n}\left(1-\frac{s k}{n}\right) d(s K(s))+o_{\mathbb{P}}(1),
$$

where $\left\{\mathbb{B}_{n}(s) n \geq 1\right\}$ is a sequence of standard Brownian bridges for $s \in(0,1)$.

Note that this result is similar to the ones in Csörgö et al. (1985) and Groeneboom et al. (2003) but under slightly different assumptions: for instance a Karamata representation is not required for $Q$ and no assumption is made on the second derivative of the kernel.

Direct computations lead to the following asymptotic normalities.

Corollary 1. Under the assumptions of Theorem 1, we have

$$
\sqrt{k}\left(\widehat{\gamma}_{n, k}^{K}-\gamma-A(n / k) \int_{0}^{1} s^{-\omega} K(s) d s\right) \stackrel{\mathcal{D}}{\longrightarrow} \mathcal{N}\left(0, \gamma^{2} \int_{0}^{1} K^{2}(s) d s\right) .
$$

In the case where $K=\underline{K}$, we find back the classical result on the Hill estimator (see e.g. Theorem 1 of de Haan and Peng, 1998).

Corollary 2. Under the assumptions of Theorem 1 and in the special case where $K=\underline{K}$, we have

$$
\sqrt{k}\left(\widehat{\gamma} \frac{K}{n, k}-\gamma-\frac{A(n / k)}{1-\omega}\right) \stackrel{\mathcal{D}}{\longrightarrow} \mathcal{N}\left(0, \gamma^{2}\right)
$$


As a consequence of Corollary 1, the Asymptotic Mean Squared Error (AMSE) of the kernel estimator $\widehat{\gamma}_{n, k}^{K}$ is given by

$$
\operatorname{AMSE}(\gamma, A(n / k), \omega)=\frac{\gamma^{2}}{k} \int_{0}^{1} K^{2}(s) d s+A^{2}\left(\frac{n}{k}\right)\left(\int_{0}^{1} s^{-\omega} K(s) d s\right)^{2} .
$$

Thus, having an estimator for $\gamma, A(n / k)$ and $\omega$ can lead to a selection criteria for $k$ :

$$
k=\arg \min A M S E(\widehat{\gamma}, \widehat{A}(n / k), \widehat{\boldsymbol{\omega}}),
$$

see also Beirlant et al. (2002), Section 4.

\subsection{Asymptotic results for the PHP estimator}

Theorem 2. Assume that $F$ satisfies $\left(\mathcal{R}_{\mathbb{U}}\right)$ with $\gamma \in(1 / 2,1)$ and suppose that the quantile function $Q$ associated to $F$ is continuously differentiable on $[0,1)$. If further $(\mathcal{K})$ holds and the sequence $k$ satisfies $k \rightarrow \infty, k / n \rightarrow 0$ and $\sqrt{k} A(n / k)=O(1)$ as $n \rightarrow \infty$, then for any $1 \leq \rho<1 / \gamma$ we have

$\frac{\sqrt{k}}{(k / n)^{1 / \rho} \mathbb{U}(n / k)}\left(\widetilde{\pi}_{n, k}^{K}(\rho)-\pi(\rho)\right) \stackrel{\mathcal{D}}{=} \sqrt{k} A\left(\frac{n}{k}\right) \mathcal{A B}_{K}(\rho, \gamma, \omega)+\mathbb{W}_{n, 1}+\mathbb{W}_{n, 2}(K)+\mathbb{W}_{n, 3}+o_{\mathbb{P}}(1)$

where

$$
\mathcal{A B}_{K}(\rho, \gamma, \omega):=\frac{\rho}{1-\rho \gamma}\left(\frac{1}{\rho \gamma+\rho \omega-1}+\frac{1}{1-\rho \gamma} \int_{0}^{1} s^{-\omega} K(s) d s\right)
$$

and

$$
\left\{\begin{array}{l}
\mathbb{W}_{n, 1}:=-\frac{\gamma}{1-\rho \gamma} \sqrt{\frac{n}{k}} \mathbb{B}_{n}(1-k / n) \\
\mathbb{W}_{n, 2}(K):=\frac{\rho \gamma}{(1-\rho \gamma)^{2}} \sqrt{\frac{n}{k}} \int_{0}^{1} s^{-1} \mathbb{B}_{n}(1-s k / n) d(s K(s)) \\
\mathbb{W}_{n, 3}:=-\frac{\rho^{-1} \int_{k / n}^{1} s^{1 / \rho-1} \mathbb{B}_{n}(1-s) Q^{\prime}(1-s) d s}{(k / n)^{1 / \rho-1 / 2} \mathbb{U}(n / k)}
\end{array}\right.
$$

Corollary 3. Under the assumptions of Theorem 2, if $\sqrt{k} A(n / k) \rightarrow \lambda \in \mathbb{R}$, we have

$$
\frac{\sqrt{k}}{(k / n)^{1 / \rho \mathbb{U}(n / k)}}\left(\widetilde{\pi}_{n, k}^{K}(\rho)-\pi(\rho)\right) \stackrel{\mathcal{D}}{\longrightarrow} \mathcal{N}\left(\lambda \mathcal{A} \mathcal{B}_{K}(\rho, \gamma, \omega), \mathcal{A} \mathcal{V}_{K}(\rho, \gamma)\right),
$$

where

$$
\mathcal{A} \mathcal{V}_{K}(\rho, \gamma)=\frac{\gamma^{2} \rho}{(1-\rho \gamma)^{2}(2 \gamma \rho+\rho-2)}+\frac{\rho^{2} \gamma^{2}}{(1-\rho \gamma)^{4}} \int_{0}^{1} K^{2}(s) d s
$$

This Corollary 3 generalizes Theorem 2 in Necir and Meraghni (2009) in case $\lambda \neq 0$ and when we use a general kernel instead of $\underline{K}$.

In view of these results, $\widetilde{\pi}_{n, k}^{K}(\rho)$ is an estimator of $\pi(\rho)$ with an asymptotic bias given by

$$
(k / n)^{1 / \rho} \mathbb{U}(n / k) A(n / k) \mathcal{A B}_{K}(\rho, \gamma, \omega) .
$$


This quantity depends on the original distribution through the functions $\mathbb{U}$ and $A$, the extremevalue parameters $\gamma$ and $\omega$, the distortion parameter $\rho$, the kernel $K$ and the ratio $k / n$ of upper order statistics. For a specific kernel, the asymptotic bias and variance can be computed. For instance, we have the following corollary if $K=\underline{K}$.

Corollary 4. Under the assumptions of Corollary 3 and in the special case where $K=\underline{K}$, we have

$\frac{\sqrt{k}}{(k / n)^{1 / \rho \mathbb{U}(n / k)}}\left(\widetilde{\pi}_{n, k}^{K}(\rho)-\pi(\rho)\right) \stackrel{\mathcal{D}}{\longrightarrow} \mathcal{N}\left(\lambda \frac{\rho \omega(\rho \gamma+\rho-1)}{(1-\omega)(\rho \gamma+\rho \omega-1)(1-\rho \gamma)^{2}}, \frac{\rho \gamma^{2}(\rho \gamma+\rho-1)^{2}}{(2 \rho \gamma+\rho-2)(1-\rho \gamma)^{4}}\right)$.

Note that this Corollary 4 corrects a mistake in the asymptotic variance of Theorem 2 in Necir and Meraghni (2009).

The goal of the next section is to propose a reduced-bias estimator of $\pi(\rho)$.

\subsection{Bias-correction for the PHP estimator}

Recall that, from Theorem 2,

$$
\widetilde{\pi}_{n, k}^{K}(\rho)-(k / n)^{1 / \rho} \mathbb{U}(n / k) A(n / k) \mathcal{A B}_{K}(\rho, \gamma, \omega)
$$

is an asymptotically unbiased estimator for $\pi(\rho)$. Note that $\gamma, \omega, \mathbb{U}(n / k)$ and $A(n / k)$ are unknown quantities that we have to estimate.

Using $\left(\mathcal{R}_{\mathbb{U}}\right)$, Feuerverger and Hall (1999) and Beirlant et al $(1999,2002)$ proposed the following exponential regression model for the log-spacings of order statistics:

$$
Z_{j, k} \sim\left(\gamma+A(n / k)\left(\frac{j}{k+1}\right)^{-\omega}\right)+\varepsilon_{j, k}, 1 \leq j \leq k,
$$

where $\varepsilon_{j, k}$ are zero-centered error terms. If we ignore the term $A(n / k)$ in $(9)$, we retrieve the Hill-type estimator $\widehat{\gamma}_{n, k}^{H}$ by taking the mean of the left-hand side of (9). By using a least-squares approach, (9) can be further exploited to propose a reduced-bias estimator for $\gamma$ in which $\omega$ is substituted by a consistent estimator $\widehat{\omega}=\widehat{\omega}_{n, k}$ (see for instance Beirlant et al, 2002) or by a canonical choice, such as $\boldsymbol{\omega}=-1$ (see e.g. Feuerverger and Hall (1999) or Beirlant et al (1999)). The least squares estimators for $\gamma$ and $A(n / k)$ are then given by

$$
\left\{\begin{array}{l}
\widehat{\gamma}_{n, k}^{\boldsymbol{L S}}(\widehat{\boldsymbol{\omega}})=\frac{1}{k} \sum_{j=1}^{k} Z_{j, k}-\frac{\widehat{A}_{n, k}^{\boldsymbol{L} \boldsymbol{S}}(\widehat{\boldsymbol{\omega}})}{1-\widehat{\boldsymbol{\omega}}} \\
\hat{A}_{n, k}^{\boldsymbol{L S}}(\widehat{\boldsymbol{\omega}})=\frac{(1-2 \widehat{\boldsymbol{\omega}})(1-\widehat{\boldsymbol{\omega}})^{2}}{\widehat{\boldsymbol{\omega}}^{2}} \frac{1}{k} \sum_{j=1}^{k}\left(\left(\frac{j}{k+1}\right)^{-\widehat{\boldsymbol{\omega}}}-\frac{1}{1-\widehat{\boldsymbol{\omega}}}\right) Z_{j, k} .
\end{array}\right.
$$

Note that $\widehat{\gamma}_{n, k}^{\boldsymbol{L} \boldsymbol{S}}(\omega)$ can be viewed as the kernel estimator $\widehat{\gamma}_{n, k}^{\left(K_{\omega}\right)}$, where for $0<u \leq 1$ :

$$
K_{\omega}(u)=\frac{1-\omega}{\omega} \underline{K}(u)+\left(1-\frac{1-\omega}{\omega}\right) \underline{K}_{\omega}(u)
$$


with $\underline{K}(u)=\mathbb{1}_{\{0<u<1\}}$ and $\underline{K}_{\omega}(u)=((1-\omega) / \omega)\left(u^{-\omega}-1\right) \mathbb{1}_{\{0<u<1\}}$, both kernels satisfying condition $(\mathcal{K})$. On the contrary $K_{\omega}$ does not satisfy statement (i) in $(\mathcal{K})$. We refer to Gomes and Martins (2004) and Gomes et al. (2007) for other techniques of bias reduction based on the estimation of the second order parameter.

We are now able to obtain a reduced-bias estimator for the PHP from (8) and using the above estimators for the different unknown quantities:

$$
\begin{aligned}
\widehat{\pi}_{n, k}^{K, \widehat{\boldsymbol{\omega}}}(\rho) & =\widetilde{\pi}_{n, k}^{K}(\rho)-\left(\frac{k}{n}\right)^{1 / \rho} X_{n-k, n} \widehat{A}_{n, k}^{\boldsymbol{L} \boldsymbol{S}}(\widehat{\boldsymbol{\omega}}) \mathcal{A} \mathcal{B}_{K}\left(\rho, \widehat{\gamma}_{n, k}^{\boldsymbol{L S}}(\widehat{\boldsymbol{\omega}}), \widehat{\boldsymbol{\omega}}\right) \\
& =\left(\frac{k}{n}\right)^{1 / \rho} X_{n-k, n}\left(\frac{1}{1-\rho \widehat{\gamma}_{n, k}^{K}}-\widehat{A}_{n, k}^{\boldsymbol{L S}}(\widehat{\boldsymbol{\omega}}) \mathcal{A} \mathcal{B}_{K}\left(\rho, \widehat{\gamma}_{n, k}^{\boldsymbol{L S}}(\widehat{\boldsymbol{\omega}}), \widehat{\boldsymbol{\omega}}\right)\right) \\
& +\sum_{j=k+1}^{n}\left(\left(\frac{j}{n}\right)^{1 / \rho}-\left(\frac{j-1}{n}\right)^{1 / \rho}\right) X_{n-j+1, n} .
\end{aligned}
$$

Our next goal is to establish, under suitable assumptions, the asymptotic normality of $\widehat{\pi}_{n, k}^{K, \widehat{\omega}}(\rho)$.

Theorem 3. Under the assumptions of Theorem 2, if $\widehat{\boldsymbol{\omega}}$ is a consistent estimator for $\omega$, then we have

$$
\frac{\sqrt{k}}{(k / n)^{1 / \rho \mathbb{U}(n / k)}}\left(\widehat{\pi}_{n, k}^{K, \widehat{\boldsymbol{\omega}}}(\rho)-\pi(\rho)\right) \stackrel{\mathcal{D}}{\longrightarrow} \mathcal{N}\left(0, \widetilde{\mathcal{A V}}_{K}(\rho, \gamma, \omega)\right)
$$

with

$$
\begin{aligned}
\widetilde{\mathcal{A V}}_{K}(\rho, \gamma, \omega) & =\mathcal{A} \mathcal{V}_{K}(\rho, \gamma)+\omega^{-2} \gamma^{2}(1-2 \omega)(1-\omega)^{2} \mathcal{A B}_{K}^{2}(\rho, \gamma, \omega) \\
& +\frac{2 \rho \gamma^{2}(1-\omega)(1-2 \omega)}{\omega^{2}(1-\rho \gamma)^{2}}\left(1-(1-\omega) \int_{0}^{1} s^{-\omega} K(s) d s\right) \mathcal{A B}_{K}(\rho, \gamma, \omega) .
\end{aligned}
$$

Let us observe that $\widehat{\pi}_{n, k}^{K, \widehat{\omega}}$ has a null asymptotic bias, which was not the case for $\widetilde{\pi}_{n, k}^{K}$ (Corollary 3 ).

Corollary 5. Under the same assumptions as in Theorem 3 and in the special case where $K=\underline{K}$, we have

$$
\frac{\sqrt{k}}{(k / n)^{1 / \rho \mathbb{U}(n / k)}}\left(\widehat{\pi}_{n, k}^{K, \widehat{\omega}}(\rho)-\pi(\rho)\right) \stackrel{\mathcal{D}}{\longrightarrow} \mathcal{N}\left(0, \frac{\rho \gamma^{2}(\rho \gamma+\rho-1)^{2}(\rho \gamma+\rho-\rho \omega-1)^{2}}{(2 \rho \gamma+\rho-2)(\rho \gamma+\rho \omega-1)^{2}(1-\rho \gamma)^{4}}\right) .
$$

Now, in the special case where $K=K_{\omega}$, as already mentioned, the estimator $\widehat{\gamma}_{n, k}^{\left(K_{\omega}\right)}$ coincides with $\widehat{\gamma}_{n, k}^{\boldsymbol{L} \boldsymbol{S}}(\omega)$. The aim of the next corollary is to establish the asymptotic normality of the resulting PHP estimator $\widehat{\pi}_{n, k}^{K_{\omega}, \widehat{\boldsymbol{\omega}}}$, denoted by $\widehat{\pi}_{n, k}^{\boldsymbol{L} \boldsymbol{S}, \widehat{\boldsymbol{\omega}}}$, when the least squares approach is adopted.

Corollary 6. Under the same assumptions as in Theorem 3 and in the special case where $K=K_{\omega}$, we have

$$
\frac{\sqrt{k}}{(k / n)^{1 / \rho \mathbb{U}(n / k)}}\left(\widehat{\pi}_{n, k}^{L \boldsymbol{S}, \widehat{\boldsymbol{\omega}}}(\rho)-\pi(\rho)\right) \stackrel{\mathcal{D}}{\longrightarrow} \mathcal{N}\left(0, \widetilde{\mathcal{A V}}_{K_{\omega}}(\rho, \gamma, \omega)\right)
$$


with

$$
\begin{aligned}
\widetilde{\mathcal{A V}}_{K_{\omega}}(\rho, \gamma, \omega) & =\frac{\rho^{2} \gamma^{2}(1-\omega)^{2}}{\omega^{2}(1-\rho \gamma)^{4}}+\frac{\rho \gamma^{2}}{(2 \rho \gamma+\rho-2)(1-\rho \gamma)^{2}} \\
& +\frac{\rho^{2} \gamma^{2}(1-2 \omega)(1-\omega)(\rho \gamma \omega+2 \rho \omega+\rho \gamma-\omega-1)}{\omega^{2}(1-\rho \gamma)^{3}(\rho \gamma+\rho \omega-1)^{2}}
\end{aligned}
$$

\section{A small simulation study}

In this section, the biased estimator $\widetilde{\pi}_{n, k}^{K}(\rho)$ and the reduced-bias one $\widehat{\pi}_{n, k}^{\boldsymbol{L} \boldsymbol{S}, \widehat{\boldsymbol{\omega}}}(\rho)$ are compared on a small simulation study. To this aim, 500 samples of size $n \in\{500,1000,1500\}$ are simulated from a Burr distribution defined as: $\bar{F}(x)=\left(1+x^{-\frac{3}{2} \omega}\right)^{1 / \omega}$. The associated extreme-value index is $\gamma=2 / 3$ and $\omega$ is the second order parameter. The aversion index $\rho$ is set to 1.1 and different values of $\omega \in\{-0.75,-1,-1.5\}$ are considered to assess its impact. The median and median squared error (MSE) of these estimators are estimated over the 500 replications. The results are displayed on Figure 2 and Figure 3. It appears on Figure 2 that the closer $\omega$ is to 0 , the more important is the bias of $\widetilde{\pi}_{n, k}^{K}(1.1)$. As a comparison, the bias of $\widehat{\pi}_{n, k}^{\boldsymbol{L} \boldsymbol{S}, \widehat{\boldsymbol{\omega}}}(1.1)$ is much smaller and almost independent of $\omega$. Unsurprisingly, all the biases decrease when $n$ increases. The effect of the bias correction on the MSE is illustrated on Figure 3. It appears that it can lead to a slight increase of the MSE when the bias is small, i.e when $\omega$ is small. However, the MSE of the reduced-bias estimator $\widehat{\pi}_{n, k}^{\boldsymbol{L}, \widehat{\boldsymbol{\omega}}}(1.1)$ is almost constant with respect to $k$, especially when the bias of $\widetilde{\pi}_{n, k}^{K}(1.1)$ is strong, i.e when $\omega$ is close to 0 . This property makes the choice of $k$ easier in practice. Similar results can be obtained with other distributions.

\section{Proofs}

Let $Y_{1}, \ldots, Y_{n}$ be independent and identically distributed random variables from the unit Pareto distribution $G$, defined as $G(y)=1-y^{-1}, y \geq 1$. For each $n \geq 1$, let $Y_{1, n} \leq \ldots \leq Y_{n, n}$ be the order statistics pertaining to $Y_{1}, \ldots, Y_{n}$. Clearly $X_{j, n} \stackrel{\mathcal{D}}{=} \mathbb{U}\left(Y_{j, n}\right), j=1, \ldots, n$. In order to use results from Csörgö et al. (1986), a probability space $(\Omega, \mathbb{A}, \mathbb{P})$ is constructed carrying a sequence $\xi_{1}, \xi_{2}, \ldots$ of independent random variables uniformly distributed on $(0,1)$ and a sequence of Brownian bridges $\mathbb{B}_{n}(s), 0 \leq s \leq 1, n=1,2 \ldots$. The resulting empirical quantile is denoted by

$$
\beta_{n}(t)=\sqrt{n}\left(t-\mathbb{V}_{n}(t)\right)
$$

where $\mathbb{V}_{n}(s)=\xi_{j, n}, \frac{j-1}{n}<s \leq \frac{j}{n}, j=1, \ldots, n$ and $\mathbb{V}_{n}(0)=0$.

\subsection{Preliminary results}

The following lemmas will be instrumental for our needs. Their proofs are postponed to Section 5 . 
Lemma 1. Let $\lambda_{n}$ be a sequence of positive numbers such that $\lambda_{n} \rightarrow 0$ and $n \lambda_{n} \rightarrow \infty$ as $n \rightarrow \infty$. Then

$$
\int_{0}^{1 /\left[\lambda_{n}(n+1)\right]}\left[\log \left(1-\mathbb{V}_{n}\left(1-s \lambda_{n}\right)\right)-\log \left(s \lambda_{n}\right)\right] d(s K(s))=o_{\mathbb{P}}\left(\frac{1}{\sqrt{n \lambda_{n}}}\right) .
$$

Lemma 2. Let $\mathbb{L}(\cdot)$ be an integrable, bounded and positive function on $(0,1)$ and $k$ a sequence of positive numbers such that $k \rightarrow \infty$ and $k / n \rightarrow 0$ as $n \rightarrow \infty$. Set $\lambda_{n}=k /(n+1)$. Then for each $\beta>0$, we have

$$
\int_{0}^{1}\left[\left(\frac{\mathbb{V}_{n}\left(s \lambda_{n}\right)}{\xi_{k+1, n}}\right)^{\beta}-s^{\beta}\right] \mathbb{L}(s) d s \stackrel{\mathbb{P}}{\longrightarrow} 0, \text { as } n \rightarrow \infty .
$$

The next two lemmas establish asymptotic expansions of the two random terms appearing in (6).

Lemma 3. Under the assumptions of Theorem 2, we have

$\frac{\sqrt{k}}{(k / n)^{1 / \rho \mathbb{U}(n / k)}}\left(\widetilde{\pi}_{n, k}^{(K, 1)}(\rho)-\pi_{n, k}^{(1)}(\rho)\right) \stackrel{\mathcal{D}}{=} \sqrt{k} A(n / k) \mathcal{A B}_{K}(\rho, \gamma, \omega)+\mathbb{W}_{n, 1}+\mathbb{W}_{n, 2}(K)+o \mathbb{P}(1)$.

Lemma 4. Under the assumptions of Theorem 2, we have

$$
\frac{\sqrt{k}}{(k / n)^{1 / \rho} \mathbb{U}(n / k)}\left(\widetilde{\pi}_{n, k}^{(2)}(\rho)-\pi_{n, k}^{(2)}(\rho)\right) \stackrel{\mathcal{D}}{=} \mathbb{W}_{n, 3}+o_{\mathbb{P}}(1)
$$

To establish Theorem 3, we need to introduce the following additional lemma. It is dedicated to the asymptotic behaviour of the least-squares estimates of $\gamma$ and $A(n / k)$.

Lemma 5. Suppose that the distribution F satisfies $\left(\mathcal{R}_{\mathbb{U}}\right)$. If $k \rightarrow \infty, k / n \rightarrow 0$ and $\sqrt{k} A(n / k)=$ $O(1)$ as $n \rightarrow \infty$, then, for any consistent estimator $\widehat{\omega}$ of $\omega$, we have

$$
\sqrt{k}\left(\widehat{\gamma}_{n, k}^{\boldsymbol{L} \boldsymbol{S}}(\widehat{\boldsymbol{\omega}})-\gamma\right) \stackrel{\mathcal{D}}{=} \gamma \sqrt{\frac{n}{k}} \int_{0}^{1} s^{-1} \mathbb{B}\left(1-\frac{s k}{n}\right) d\left(s K_{\omega}(s)\right)+o_{\mathbb{P}}(1)
$$

and

$$
\sqrt{k}\left(\widehat{A}_{n, k}^{\boldsymbol{L S}}(\widehat{\boldsymbol{\omega}})-A(n / k)\right) \stackrel{\mathcal{D}}{=} \gamma(1-\omega) \sqrt{\frac{n}{k}} \int_{0}^{1} s^{-1} \mathbb{B}\left(1-\frac{s k}{n}\right) d\left(s\left(\underline{K}(s)-K_{\omega}(s)\right)\right)+o_{\mathbb{P}}(1) .
$$

Last lemma is a direct consequence of Karamata's theorem (see Proposition 1.5.8 in Bingham et al., 1987).

Lemma 6. Let $\ell$ be a slowly varying function at 0 . Then, for all $\alpha>1$,

$$
\lim _{s \rightarrow 0} \frac{1}{s^{1-\alpha} \ell(s)} \int_{s}^{1} t^{-\alpha} \ell(t) d t=\frac{1}{\alpha-1} .
$$

\subsection{Proofs of the main results}

Proof of Theorem 1. As already mentioned, our assumptions $(\mathcal{K})$ are similar to those in Csörgö et al. (1985). In particular our kernel estimator $\widehat{\gamma}_{n, k}^{K}$ is a particular example of their estimate

$$
a_{n}^{-1}=\sum_{j=1}^{n} \frac{1}{n \lambda_{n}} \widetilde{K}\left(\frac{j}{n \lambda_{n}}\right) Z_{j, k}\left(\int_{0}^{\frac{1}{\lambda_{n}}} \widetilde{K}(u) d u\right)^{-1}
$$


where $\lambda_{n}=\frac{k+1}{n}, \widetilde{K}=K$ on $(0,1]$ and 0 otherwise. Consequently, the weak consistency of $\widehat{\gamma}_{n, k}^{K}$ yields

$$
\begin{aligned}
\widehat{\gamma}_{n, k}^{K} & =\sum_{j=1}^{n} \frac{1}{n \lambda_{n}} \widetilde{K}\left(\frac{j}{n \lambda_{n}}\right) Z_{j, k}+O_{\mathbb{P}}\left(\frac{1}{k}\right) \\
& =\int_{0}^{1 / \lambda_{n}} \log Q_{n}\left(1-s \lambda_{n}\right) d(s \widetilde{K}(s))+O_{\mathbb{P}}\left(\frac{1}{k}\right) \\
& =\int_{0}^{1} \log Q_{n}\left(1-s \lambda_{n}\right) d(s K(s))+O_{\mathbb{P}}\left(\frac{1}{k}\right) \\
& \stackrel{\mathcal{D}}{=} \int_{0}^{1}\left[\log Q_{n}\left(1-s \lambda_{n}\right)-\log Q\left(1-\xi_{k+1, n}\right)\right] d(s K(s))+O_{\mathbb{P}}\left(\frac{1}{k}\right),
\end{aligned}
$$

by Lemma 2(i) in Csörgő et al. (1985). Finally, it follows that

$$
\widehat{\gamma}_{n, k}^{K} \stackrel{\mathcal{D}}{=} \int_{0}^{1}\left[\log Q\left(1-\mathbb{V}_{n}\left(s \lambda_{n}\right)\right)-\log Q\left(1-\xi_{k+1, n}\right)\right] d(s K(s))+O_{\mathbb{P}}\left(\frac{1}{k}\right) .
$$

Note that, according to de Haan and Ferreira (2006, page 74 ), the second-order condition $\left(\mathcal{R}_{\mathbb{U}}\right)$ implies that for a possibly different function $A_{0}$, with $A_{0}(t) \sim A(t), t \rightarrow \infty$, and for each $\delta>0$, there exists a $t_{0}(\delta)$ such that for $t \geq t_{0}(\delta), x \geq 1$,

$$
\left|\frac{\log \mathbb{U}(t x)-\log \mathbb{U}(t)-\gamma \log x}{A_{0}(t)}-\frac{x^{\omega}-1}{\omega}\right| \leq \delta x^{\omega+\delta} .
$$

Thus,

$$
\begin{aligned}
\widehat{\gamma}_{n, k}^{K}= & \gamma \int_{0}^{1} \log \left(\frac{\xi_{k+1, n}}{\mathbb{V}_{n}\left(s \lambda_{n}\right)}\right) d(s K(s))+A_{0}\left(\frac{1}{\xi_{k+1, n}}\right) \int_{0}^{1} \frac{\left(\frac{\xi_{k+1, n}}{\mathbb{V}_{n}\left(s \lambda_{n}\right)}\right)^{\omega}-1}{\omega} d(s K(s)) \\
& +o_{\mathbb{P}}\left(A_{0}\left(\frac{1}{\xi_{k+1, n}}\right)\right) \int_{0}^{1}\left(\frac{\xi_{k+1, n}}{\mathbb{V}_{n}\left(s \lambda_{n}\right)}\right)^{\omega+\delta} d(s K(s))+O_{\mathbb{P}}\left(\frac{1}{k}\right) \\
=: & A_{n 1}+A_{n 2}+A_{n 3}+O_{\mathbb{P}}\left(\frac{1}{k}\right)
\end{aligned}
$$

and each term is studied separately.

Term $A_{n 1}$ : Again, by Lemma 2(i) in Csörgö et al. (1985), we have

$$
A_{n 1}=-\gamma \int_{0}^{1 / \lambda_{n}} \log \mathbb{V}_{n}\left(s \lambda_{n}\right) d(s \widetilde{K}(s)) \stackrel{\mathcal{D}}{=} \gamma-\gamma \int_{0}^{1}\left[\log \mathbb{V}_{n}\left(s \lambda_{n}\right)-\log \left(s \lambda_{n}\right)\right] d(s K(s)),
$$

by Lemma 2(ii) in Csörgő et al. (1985). It follows that

$$
\begin{aligned}
A_{n 1} & \stackrel{\mathcal{D}}{=} \gamma-\gamma \int_{0}^{1}\left[\log \left(1-\mathbb{V}_{n}\left(1-s \lambda_{n}\right)\right)-\log \left(s \lambda_{n}\right)\right] d(s K(s)) \\
& \stackrel{\mathcal{D}}{=} \gamma-\gamma \int_{1 /\left[\lambda_{n}(n+1)\right]}^{1}\left[\log \left(1-\mathbb{V}_{n}\left(1-s \lambda_{n}\right)\right)-\log \left(s \lambda_{n}\right)\right] d(s K(s))+o_{\mathbb{P}}\left(\frac{1}{\sqrt{n \lambda_{n}}}\right),
\end{aligned}
$$


from Lemma 1. A straightforward expansion of the integral yields

$$
\begin{aligned}
A_{n 1} \stackrel{\mathcal{D}}{=} & \gamma-\gamma \int_{1 /\left[\lambda_{n}(n+1)\right]}^{1 / \lambda_{n}}\left[\log \left(1-\mathbb{V}_{n}\left(1-s \lambda_{n}\right)\right)-\log \left(s \lambda_{n}\right)+\frac{\mathbb{B}_{n}\left(1-s \lambda_{n}\right)}{s \lambda_{n} \sqrt{n}}\right] d(s \widetilde{K}(s)) \\
& +\gamma \int_{1 /\left[\lambda_{n}(n+1)\right]}^{1 / \lambda_{n}} \frac{\mathbb{B}_{n}\left(1-s \lambda_{n}\right)}{s \lambda_{n} \sqrt{n}} d(s \widetilde{K}(s))+o_{\mathbb{P}}\left(\frac{1}{\sqrt{n \lambda_{n}}}\right) \\
\stackrel{\mathcal{D}}{=} & \gamma+\gamma \int_{1 /\left[\lambda_{n}(n+1)\right]}^{1 / \lambda_{n}} \frac{\mathbb{B}_{n}\left(1-s \lambda_{n}\right)}{s \lambda_{n} \sqrt{n}} d(s \widetilde{K}(s))+o_{\mathbb{P}}\left(\frac{1}{\sqrt{n \lambda_{n}}}\right)
\end{aligned}
$$

by Lemma 12 in Csörgő et al. (1985). Finally, Lemma 8 in Csörgő et al. (1985) entails that

$$
A_{n 1} \stackrel{\mathcal{D}}{=} \gamma+\gamma \int_{0}^{1} \frac{\mathbb{B}_{n}\left(1-s \lambda_{n}\right)}{s \lambda_{n} \sqrt{n}} d(s K(s))+o \mathbb{P}\left(\frac{1}{\sqrt{n \lambda_{n}}}\right)
$$

under our assumptions on $k$ and $(\mathcal{K})$.

Term $A_{n 2}$ : Let $\mathbb{L}(s)=d(s K(s)) / d s=\mathbb{L}^{+}(s)-\mathbb{L}^{-}(s)$ where $\mathbb{L}^{ \pm}(s)$ are positive and bounded functions. We have

$$
\begin{aligned}
A_{n 2} & =\frac{1}{\omega} A_{0}\left(\frac{1}{\xi_{k+1, n}}\right) \int_{0}^{1}\left(\frac{\mathbb{V}_{n}\left(s \lambda_{n}\right)}{\xi_{k+1, n}}\right)^{-\omega} \mathbb{L}(s) d s \\
& =\frac{1}{\omega} A_{0}\left(\frac{1}{\xi_{k+1, n}}\right)\left\{\int_{0}^{1}\left(\frac{\mathbb{V}_{n}\left(s \lambda_{n}\right)}{\xi_{k+1, n}}\right)^{-\omega} \mathbb{L}^{+}(s) d s-\int_{0}^{1}\left(\frac{\mathbb{V}_{n}\left(s \lambda_{n}\right)}{\xi_{k+1, n}}\right)^{-\omega} \mathbb{L}^{-}(s) d s\right\} \\
& =\frac{1}{\omega} A_{0}\left(\frac{1}{\xi_{k+1, n}}\right)\left(\int_{0}^{1} s^{-\omega} \mathbb{L}^{+}(s) d s-\int_{0}^{1} s^{-\omega} \mathbb{L}^{-}(s) d s+o_{\mathbb{P}}(1)\right),
\end{aligned}
$$

by Lemma 2. An integration by parts yields

$$
\begin{aligned}
A_{n 2} & =\frac{1}{\omega} A_{0}\left(\frac{1}{\xi_{k+1, n}}\right)\left(\int_{0}^{1} s^{-\omega} \mathbb{L}(s) d s+o_{\mathbb{P}}(1)\right) \\
& =A_{0}\left(\frac{1}{\xi_{k+1, n}}\right)\left(\int_{0}^{1} s^{-\omega} K(s) d s+o_{\mathbb{P}}(1)\right) \\
& =A_{0}\left(\frac{n}{k}\right)\left(\int_{0}^{1} s^{-\omega} K(s) d s+o_{\mathbb{P}}(1)\right)
\end{aligned}
$$

since $A_{0}$ is regularly varying at infinity.

Term $A_{n 3}$ : Similarly as for the preceding term, by Lemma 2 with $\beta=-\omega-\delta>0$, and under our assumptions

$$
A_{n 3}=o_{\mathbb{P}}\left(A\left(\frac{n}{k}\right)\right) .
$$

Finally, combining (14), (15) and (16), Theorem 1 follows.

Proof of Theorem 2. Combining Lemmas 3 and 4, we get

$\frac{\sqrt{k}}{(k / n)^{1 / \rho} \mathbb{U}(n / k)}\left(\widetilde{\pi}_{n, k}^{K}(\rho)-\pi(\rho)\right) \stackrel{\mathcal{D}}{=} \sqrt{k} A\left(\frac{n}{k}\right) \mathcal{A B}_{K}(\rho, \gamma, \omega)+\mathbb{W}_{n, 1}+\mathbb{W}_{n, 2}(K)+\mathbb{W}_{n, 3}+o_{\mathbb{P}}(1)$.

Theorem 2 is thus established. 
Proof of Corollary 3. From Theorem 2, we only have to compute the asymptotic variance of the limiting process. The computations are tedious but quite direct. We only give below the main arguments. Remark that

$$
\begin{aligned}
\frac{J_{\alpha}(s)}{s^{\alpha} Q(1-s)}:=\frac{\int_{s}^{1} t^{\alpha} Q^{\prime}(1-t) d t}{s^{\alpha} Q(1-s)} & =1+\alpha \frac{\int_{s}^{1} t^{\alpha-1} Q(1-t) d t}{s^{\alpha} Q(1-s)} \\
& =1+\alpha \frac{\int_{s}^{1} t^{\alpha-\gamma-1} \ell(t) d t}{s^{\alpha-\gamma} \ell(s)} \longrightarrow \frac{\gamma}{\gamma-\alpha} \quad \text { if } \gamma>\alpha
\end{aligned}
$$

by Lemma 6 and using the fact that $Q(1-$.) is regularly varying at 0 with index $-\gamma$ under $(3)$, that is, $Q(1-s)=s^{-\gamma} \ell(s)$ with $\ell$ a slowly varying function at 0 . This allows us to prove in particular that

$$
\frac{\int_{s}^{1} J_{\frac{1}{\rho}-1}^{2}(t) d t}{s J_{\frac{1}{\rho}-1}^{2}(s)} \longrightarrow \frac{\rho}{2 \gamma \rho-2+\rho} \text { as } s \rightarrow 0 .
$$

Also, from Proposition 1.3.6 in Bingham et al. (1987), it follows that

$$
\begin{gathered}
\forall \varepsilon>0, \quad x^{-\varepsilon} \ell(x) \quad \longrightarrow \quad \infty \text { as } x \rightarrow 0 \\
\forall \delta>0, \quad x^{\delta} \ell(x) \quad \longrightarrow \quad 0 \text { as } x \rightarrow 0 .
\end{gathered}
$$

Thus, choosing $0<\delta<-\gamma+\frac{1}{\rho}$ and $0<\varepsilon<\gamma-\frac{1}{\rho}+\frac{1}{2}$ entails

$$
\begin{aligned}
0 \leq s\left(\frac{J_{\frac{1}{\rho}}(s)}{s^{\frac{1}{\rho}} Q(1-s)}\right)^{2} & =s\left(1+\frac{1}{\rho} \frac{\int_{s}^{1} t^{\frac{1}{\rho}-\gamma-1} \ell(t) d t}{s^{\frac{1}{\rho}-\gamma} \ell(s)}\right)^{2} \\
& \leq s\left(1+C s^{\gamma-\frac{1}{\rho}-\varepsilon}\right)^{2}=O\left(s^{1+2\left[\gamma-\frac{1}{\rho}-\varepsilon\right]}\right)=o(1)
\end{aligned}
$$

under our assumptions, where $C$ is a suitable constant.

Proof of Theorem 3. According to Theorem 2 and (12), we have

$$
\frac{\sqrt{k}\left(\widehat{\pi}_{n, k}^{K, \widehat{\omega}}(\rho)-\pi(\rho)\right)}{(k / n)^{1 / \rho} \mathbb{U}(n / k)} \stackrel{\mathcal{D}}{=} \mathbb{W}_{n, 1}+\mathbb{W}_{n, 2}(K)+\mathbb{W}_{n, 3}+\mathbb{W}_{n, 4}(K)+o_{\mathbb{P}}(1),
$$

where

$$
\begin{aligned}
& \mathbb{W}_{n, 4}(K):=\sqrt{k}\left(A(n / k) \mathcal{A} \mathcal{B}_{K}(\rho, \gamma, \omega)-\widehat{A}_{n, k}^{\boldsymbol{L S}}(\widehat{\boldsymbol{\omega}}) \mathcal{A B}_{K}\left(\rho, \widehat{\gamma}_{n, k}^{\boldsymbol{L S}}(\widehat{\boldsymbol{\omega}}), \widehat{\boldsymbol{\omega}}\right) \frac{X_{n-k, n}}{\mathbb{U}(n / k)}\right) \\
& =-\mathcal{A B}_{K}(\rho, \gamma, \omega) \sqrt{k}\left(\widehat{A}_{n, k}^{L S}(\widehat{\boldsymbol{\omega}})-A(n / k)\right) \\
& -\sqrt{k} \widehat{A}_{n, k}^{\boldsymbol{L} \boldsymbol{S}}(\widehat{\boldsymbol{\omega}})\left(\mathcal{A B}_{K}\left(\rho, \widehat{\gamma}_{n, k}^{\boldsymbol{L} \boldsymbol{S}}(\widehat{\boldsymbol{\omega}}), \widehat{\boldsymbol{\omega}}\right)-\mathcal{A B}_{K}(\rho, \gamma, \omega)\right) \\
& -\sqrt{k} \widehat{A}_{n, k}^{\boldsymbol{L} \boldsymbol{S}}(\widehat{\boldsymbol{\omega}}) \mathcal{A} \mathcal{B}_{K}\left(\rho, \widehat{\gamma}_{n, k}^{\boldsymbol{L} \boldsymbol{S}}(\widehat{\boldsymbol{\omega}}), \widehat{\boldsymbol{\omega}}\right)\left(\frac{X_{n-k, n}}{\mathbb{U}(n / k)}-1\right) \\
& \stackrel{\mathcal{D}}{=}-\mathcal{A B}_{K}(\rho, \gamma, \omega) \gamma(1-\omega) \sqrt{\frac{n}{k}} \int_{0}^{1} s^{-1} \mathbb{B}_{n}\left(1-\frac{s k}{n}\right) d\left(s\left(\underline{K}(s)-K_{\omega}(s)\right)\right)+o_{\mathbb{P}}(1),
\end{aligned}
$$

by Lemma 5 , using the consistency and the inequality $\left|\frac{e^{x}-1}{x}-1\right| \leq e^{|x|}-1$ for all $x \in \mathbb{R}$. Moreover, direct computations lead to the desired asymptotic variance which ends the proof of Theorem 3. 
Proof of Corollary 6. Recall that $K_{\omega}$ does not satisfy condition $(\mathcal{K})$ but it can be rewritten as (11) with both $\underline{K}$ and $\underline{K}_{\omega}$ satisfying $(\mathcal{K})$. So, following the lines of proof of Theorem 3 and using Lemma 5, Corollary 6 follows.

\section{$5 \quad$ Proofs of auxiliary results}

Proof of Lemma 1. Our aim is to study

$$
\begin{aligned}
Q_{n} & :=\int_{0}^{1 /\left[\lambda_{n}(n+1)\right]}\left[\log \left(1-\xi_{n, n}\right)+\log (n+1)\right] d(s K(s))-\int_{0}^{1 /\left[\lambda_{n}(n+1)\right]} \log \left(s \lambda_{n}(n+1)\right) d(s K(s)) \\
& =: Q_{n}^{(1)}+Q_{n}^{(2)} .
\end{aligned}
$$

Recall that $\lim _{n \rightarrow \infty} \mathbb{P}\left(-\log \left(1-\xi_{n, n}\right)-\log (n+1) \leq x\right)=e^{-e^{-x}}$ since an exponential variable is in the Gumbel domain of attraction. Thus,

$$
\sqrt{n \lambda_{n}} Q_{n}^{(1)}=\sqrt{n \lambda_{n}} O_{\mathbb{P}}(1) \int_{0}^{1 /\left[\lambda_{n}(n+1)\right]} d(s K(s))=o_{\mathbb{P}}(1),
$$

by Lemma 1(i) in Csörgö et al. (1985). Similarly, by Lemmas 1(i) and 2(ii) in Csörgő et al. (1985):

$$
\begin{aligned}
\sqrt{n \lambda_{n}} Q_{n}^{(2)} & =-\sqrt{n \lambda_{n}} \int_{0}^{1 /\left[\lambda_{n}(n+1)\right]} \log \left(s \lambda_{n}(n+1)\right) d(s K(s)) \\
& =\sqrt{n \lambda_{n}} \int_{0}^{1 /\left[\lambda_{n}(n+1)\right]} K(s) d s \\
& =o_{\mathbb{P}}(1) .
\end{aligned}
$$

This achieves the proof of Lemma 1.

Proof of Lemma 2. It is a direct consequence of Lemma 3.2 in Groeneboom et al (2003).

Proof of Lemma 3. Note that $\widetilde{\pi}_{n, k}^{(K, 1)}(\rho)$ can be rewritten as follows

$$
\tilde{\pi}_{n, k}^{(K, 1)}(\rho)=\frac{(k / n)^{1 / \rho}}{1-\rho \widehat{\gamma}_{n, k}^{K}} \mathbb{U}\left(Y_{n-k, n}\right) .
$$

As a consequence, the following expansion holds:

$$
\frac{\sqrt{k}}{(k / n)^{1 / \rho} \mathbb{U}(n / k)}\left(\widetilde{\pi}_{n, k}^{(K, 1)}(\rho)-\pi_{n, k}^{(1)}(\rho)\right)=\sum_{j=1}^{4} T_{n, j},
$$

where

$$
\begin{aligned}
T_{n, 1} & :=\frac{\sqrt{k}}{1-\rho \widehat{\gamma}_{n, k}^{K}}\left[\frac{\mathbb{U}\left(Y_{n-k, n}\right)}{\mathbb{U}(n / k)}-\left(\frac{k}{n} Y_{n-k, n}\right)^{\gamma}\right], \\
T_{n, 2} & :=\frac{\sqrt{k}}{1-\rho \widehat{\gamma}_{n, k}^{K}}\left[\left(\frac{k}{n} Y_{n-k, n}\right)^{\gamma}-1\right], \\
T_{n, 3} & :=\frac{\rho}{\left(1-\rho \widehat{\gamma}_{n, k}^{K}\right)(1-\rho \gamma)} \sqrt{k}\left(\widehat{\gamma}_{n, k}^{K}-\gamma\right), \\
T_{n, 4} & :=\frac{\sqrt{k}}{(k / n)^{1 / \rho} \mathbb{U}(n / k)}\left[\frac{(k / n)^{1 / \rho}}{1-\rho \gamma} \mathbb{U}(n / k)-\pi_{n, k}^{(1)}(\rho)\right] .
\end{aligned}
$$


We study each term separately.

Term $T_{n, 1}$. According to de Haan and Ferreira (2006, p. 60 and Theorem 2.3.9, p. 48), for any

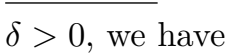

$$
\frac{\mathbb{U}\left(Y_{n-k, n}\right)}{\mathbb{U}(n / k)}-\left(\frac{k}{n} Y_{n-k, n}\right)^{\gamma}=A_{0}\left(\frac{n}{k}\right)\left\{\left(\frac{k}{n} Y_{n-k, n}\right)^{\gamma} \frac{\left(\frac{k}{n} Y_{n-k, n}\right)^{\omega}-1}{\omega}+o_{\mathbb{P}}(1)\left(\frac{k}{n} Y_{n-k, n}\right)^{\gamma+\omega \pm \delta}\right\},
$$

where $A_{0}(t) \sim A(t)$ as $t \rightarrow \infty$.

Thus, since $k Y_{n-k, n} / n=1+o_{\mathbb{P}}(1)$ and $\widehat{\gamma}_{n, k}^{K} \stackrel{\mathbb{P}}{\rightarrow} \gamma$, it readily follows that

$$
T_{n, 1}=o_{\mathbb{P}}(1)
$$

$\underline{\text { Term } T_{n, 2}}$. The equality $Y_{n-k, n} \stackrel{\mathcal{D}}{=}\left(1-\xi_{n-k, n}\right)^{-1}$ yields

$$
\begin{aligned}
\sqrt{k}\left[\left(\frac{k}{n} Y_{n-k, n}\right)^{\gamma}-1\right] & \stackrel{\mathcal{D}}{=} \sqrt{k}\left(\left(\frac{n}{k}\left(1-\xi_{n-k, n}\right)\right)^{-\gamma}-1\right) \\
& =-\gamma \sqrt{k}\left(\frac{n}{k}\left(1-\xi_{n-k, n}\right)-1\right)\left(1+o_{\mathbb{P}}(1)\right) \quad \text { by a Taylor expansion } \\
& =-\gamma \sqrt{\frac{n}{k}} \beta_{n}\left(1-\frac{k}{n}\right)\left(1+o_{\mathbb{P}}(1)\right) \\
& =-\gamma \sqrt{\frac{n}{k}}\left(\mathbb{B}_{n}\left(1-\frac{k}{n}\right)+O_{\mathbb{P}}\left(n^{-\nu}\right)\left(\frac{k}{n}\right)^{1 / 2-\nu}\right)\left(1+o_{\mathbb{P}}(1)\right),
\end{aligned}
$$

for $0 \leq \nu<1 / 2$, by Csörgő et al. (1986). Thus, using again that $\widehat{\gamma}_{n, k}^{K} \stackrel{\mathbb{P}}{\rightarrow} \gamma$, it follows that

$$
T_{n, 2} \stackrel{\mathcal{D}}{=}-\frac{\gamma}{1-\rho \gamma} \sqrt{\frac{n}{k}} \mathbb{B}_{n}\left(1-\frac{k}{n}\right)\left(1+o_{\mathbb{P}}(1)\right)=\mathbb{W}_{n, 1}+o_{\mathbb{P}}(1) .
$$

Term $T_{n, 3}$. According to Theorem 1 and by the consistency in probability of $\widehat{\gamma}_{n, k}^{K}$, we have

$$
\begin{aligned}
T_{n, 3} & \stackrel{\mathcal{D}}{=} \frac{\rho}{(1-\rho \gamma)^{2}}\left\{\sqrt{k} A\left(\frac{n}{k}\right) \int_{0}^{1} s^{-\omega} K(s) d s+\gamma \sqrt{\frac{n}{k}} \int_{0}^{1} s^{-1} \mathbb{B}_{n}\left(1-\frac{k}{n}\right) d(s K(s))\right\}+o_{\mathbb{P}}(1) \\
& =\frac{\rho}{(1-\rho \gamma)^{2}} \sqrt{k} A\left(\frac{n}{k}\right) \int_{0}^{1} s^{-\omega} K(s) d s+\mathbb{W}_{n, 2}(K)+o_{\mathbb{P}}(1)
\end{aligned}
$$

Term $T_{n, 4}$. A change of variables and an integration by parts yield

$$
\begin{aligned}
T_{n, 4} & =\sqrt{k}\left\{\frac{1}{1-\rho \gamma}-\frac{1}{\rho} \int_{1}^{\infty} x^{-1-1 / \rho} \frac{\mathbb{U}(n x / k)}{\mathbb{U}(n / k)} d x\right\} \\
& =-\frac{1}{\rho} \sqrt{k} \int_{1}^{\infty} x^{-1-1 / \rho}\left(\frac{\mathbb{U}(n x / k)}{\mathbb{U}(n / k)}-x^{\gamma}\right) d x .
\end{aligned}
$$

Thus, Theorem 2.3.9 in de Haan and Ferreira (2006) entails that, for $\gamma \in(1 / 2,1)$ and $1 \leq \rho<1 / \gamma$,

$$
\begin{aligned}
T_{n, 4} & =-\frac{1}{\rho} \sqrt{k} A_{0}\left(\frac{n}{k}\right) \int_{1}^{\infty} x^{\gamma-1-1 / \rho} \frac{x^{\omega}-1}{\omega} d x(1+o(1)) \\
& =\sqrt{k} A\left(\frac{n}{k}\right) \frac{\rho}{(1-\rho \gamma)(\rho \gamma+\rho \omega-1)}\left(1+o_{\mathbb{P}}(1)\right) .
\end{aligned}
$$

Combining (18)-(21), Lemma 3 follows. 
Proof of Lemma 4. The proof follows from the proof of Theorem 2 in Necir and Meraghni (2009).

Proof of Lemma 5. Note that the first quantity of interest can be expanded as

$$
\begin{aligned}
& \sqrt{k}\left(\widehat{\gamma}_{n, k}^{\boldsymbol{L} \boldsymbol{S}}(\widehat{\boldsymbol{\omega}})-\gamma\right)=\sqrt{k}\left(\widehat{\gamma}_{n, k}^{\boldsymbol{L} \boldsymbol{S}}(\widehat{\boldsymbol{\omega}})-\widehat{\gamma}_{n, k}^{\boldsymbol{L} \boldsymbol{S}}(\omega)\right)+\sqrt{k}\left(\widehat{\gamma}_{n, k}^{\boldsymbol{L} \boldsymbol{S}}(\omega)-\gamma\right) \\
& =\sqrt{k} \frac{1}{k} \sum_{j=1}^{k}\left\{K_{\widehat{\omega}}^{*}\left(\frac{j}{k+1}\right)-K_{\omega}^{*}\left(\frac{j}{k+1}\right)\right\} Z_{j, k}+\sqrt{k}\left(\widehat{\gamma}_{n, k}^{\boldsymbol{L S}}(\omega)-\gamma\right)
\end{aligned}
$$

where

$$
K_{\omega}^{*}(x)=\frac{(1-\omega)^{2}}{\omega^{2}}-\frac{(1-\omega)(1-2 \omega)}{\omega^{2}} x^{-\omega} .
$$

Thus, according to the proof of Theorem 3.2 of Beirlant et al. (2002), we have

$$
\sqrt{k}\left(\widehat{\gamma}_{n, k}^{\boldsymbol{L} \boldsymbol{S}}(\widehat{\boldsymbol{\omega}})-\gamma\right)=\sqrt{k}\left(\widehat{\gamma}_{n, k}^{\boldsymbol{L} \boldsymbol{S}}(\omega)-\gamma\right)+o_{\mathbb{P}}(1) .
$$

Recall now that $\widehat{\gamma}_{n, k}^{\boldsymbol{L} \boldsymbol{S}}(\omega)$ can be viewed as the kernel estimator $\widehat{\gamma}_{n, k}^{K_{\omega}}$. Consequently,

$$
\widehat{\gamma}_{n, k}^{L S}(\omega)=\frac{1-\omega}{\omega} \widehat{\gamma}_{n, k}^{K}+\left(1-\frac{1-\omega}{\omega}\right) \widehat{\gamma}_{n, k}^{\frac{K}{\omega}}
$$

and Theorem 1 yields

$$
\begin{aligned}
\sqrt{k}\left(\widehat{\gamma} \frac{K}{n, k}-\gamma-\frac{A(n / k)}{1-\omega}\right) & \stackrel{\mathcal{D}}{=} \gamma \sqrt{\frac{n}{k}} \int_{0}^{1} s^{-1} \mathbb{B}\left(1-\frac{s k}{n}\right) d(s \underline{K}(s))+o_{\mathbb{P}}(1), \\
\sqrt{k}\left(\widehat{\gamma}_{n, k}-\gamma-\frac{A(n / k)}{1-2 \omega}\right) & \stackrel{\mathcal{D}}{=} \gamma \sqrt{\frac{n}{k}} \int_{0}^{1} s^{-1} \mathbb{B}\left(1-\frac{s k}{n}\right) d\left(s \underline{K}_{\omega}(s)\right)+o_{\mathbb{P}}(1) .
\end{aligned}
$$

Combining the last two equalities leads to the first part of Lemma 5.

Focussing on the second part, (10) entails

$$
\widehat{A}_{n, k}^{\boldsymbol{L} \boldsymbol{S}}(\widehat{\boldsymbol{\omega}})=(1-\widehat{\boldsymbol{\omega}})\left(\widehat{\gamma}_{n, k}^{\frac{K}{\gamma}}-\widehat{\gamma}_{n, k}^{\boldsymbol{L} \boldsymbol{S}}(\widehat{\boldsymbol{\omega}})\right) .
$$

Thus,

$$
\begin{aligned}
\sqrt{k}\left(\widehat{A}_{n, k}^{\boldsymbol{L} \boldsymbol{S}}(\widehat{\boldsymbol{\omega}})-A(n / k)\right) & =(1-\widehat{\boldsymbol{\omega}}) \sqrt{k}\left(\widehat{\gamma}_{n, k}^{K}-\gamma-\frac{A(n / k)}{1-\omega}\right)-(1-\widehat{\boldsymbol{\omega}}) \sqrt{k}\left(\widehat{\gamma}_{n, k}^{\boldsymbol{L S}}(\widehat{\boldsymbol{\omega}})-\gamma\right) \\
& +\sqrt{k} A\left(\frac{n}{k}\right)\left(\frac{1-\widehat{\boldsymbol{\omega}}}{1-\omega}-1\right)
\end{aligned}
$$

which leads to the desired result.

\section{Acknowledgements}

The first author acknowledges support from AIRES-Sud (AIRES-Sud is a program from the French Ministry of Foreign and European Affairs, implemented by the "Institut de Recherche pour le Développement", IRD-DSF) and from the "Ministère de la Recherche Scientifique" of Sénégal. 


\section{References}

[1] Beirlant, J., Dierckx, G., Goegebeur, M., Matthys, G. (1999). Tail index estimation and an exponential regression model, Extremes, 2, 177-200.

[2] Beirlant, J., Dierckx, G., Guillou, A., Starica, C. (2002). On exponential representations of log-spacings of extreme order statistics, Extremes, 5, 157-180.

[3] Bingham, N.H., Goldie, C.M., Teugels, J.L. (1987). Regular variation, Cambridge.

[4] Centeno, M.L., Andrade e Silva, J. (2005). Applying the proportional hazard premium calculation principle, Astin Bulletin, 35, 409-425.

[5] Csörgő, M., Csörgő, S., Horváth, L., Mason, D.M. (1986). Weighted empirical and quantile processes, Annals of Probability, 14, 31-85.

[6] Csörgo, S., Deheuvels, P., Mason, D.M. (1985). Kernel estimates of the tail index of a distribution, Annals of Statistics, 13, 1050-1077.

[7] de Haan, L., Ferreira, A. (2006). Extreme value theory: an introduction, Springer.

[8] de Haan, L., Peng, L. (1998). Comparison of tail index estimators, Statistica Neerlandica, 52, 60-70.

[9] Feuerverger, A., Hall, P. (1999). Estimating a tail exponent by modelling departure from a Pareto distribution, Annals of Statistics, 27, 760-781.

[10] Geluk, J.L., de Haan, L. (1987). Regular variation, extensions and Tauberian theorems, CWI tract 40, Center for Mathematics and Computer Science, P.O. Box 4079, 1009 AB Amsterdam, The Netherlands.

[11] Gomes, M.I., Martins, M.J. (2004). Bias reduction and explicit semi-parametric estimation of the tail index, Journal of Statistical Planning and Inference, 124, 361-378.

[12] Gomes, M.I., Martins, M.J., Neves, M. (2007). Improving second order reduced bias extreme value index estimator, REVSTAT - Statistical Journal, 5(2), 177-207, 2007.

[13] Goovaerts, M.J., de Vlyder, F., Haezendonck, J. (1984). Insurance premiums, theory and applications, North Holland, Amsterdam.

[14] Groeneboom, P., Lopuhä̈, H. P., de Wolf, P. P. (2003). Kernel-type estimators for the extreme value index, Annals of Statistics, 31, 1956-1995.

[15] Hill, B. M. (1975). A simple approach to inference about the tail of a distribution, Annals of Statistics, 3, 1136-1174. 
[16] Jones, B. L., Zitikis, R. (2003). Empirical estimation of risk measures and related quantities, North American Actuarial Journal, 7, 44-54.

[17] Necir, A., Boukhetala, K. (2004). Estimating the risk adjusted premium of the largest reinsurance covers, In Antoch. Jaromir (Ed.), Proceeding of Computational Statistics, Physica-Verlag, Springer.

[18] Necir, A., Meraghni, D. (2009). Empirical estimation of the proportional hazard premium for heavy-tailed claim amounts, Insurance: Mathematics and Economics, 45, 49-58.

[19] Necir, A., Meraghni, D., Meddi, F. (2007). Statistical estimate of the proportional hazard premium of loss, Scandinavian Actuarial Journal, 3, 147-161.

[20] Vandewalle, B., Beirlant, J. (2006). On univariate extreme value statistics and the estimation of reinsurance premiums, Insurance: Mathematics and Economics, 38, 441-459.

[21] Wang, S. (1995). Insurance pricing and increased limits ratemaking by proportional hazard transforms, Insurance: Mathematics and Economics, 17, 43-54.

[22] Wang, S. (1996). Premium calculation by transforming the layer premium density, Astin Bulletin, 26, 71-92.

[23] Weissman, I., (1978). Estimation of parameters and larges quantiles based on the $k$ largest observations, Journal of American Statistical Association, 73, 812-815.

[24] Yaari, M.E. (1987). The dual theory of choice under risk, Econometrica, 55, 95-115. 

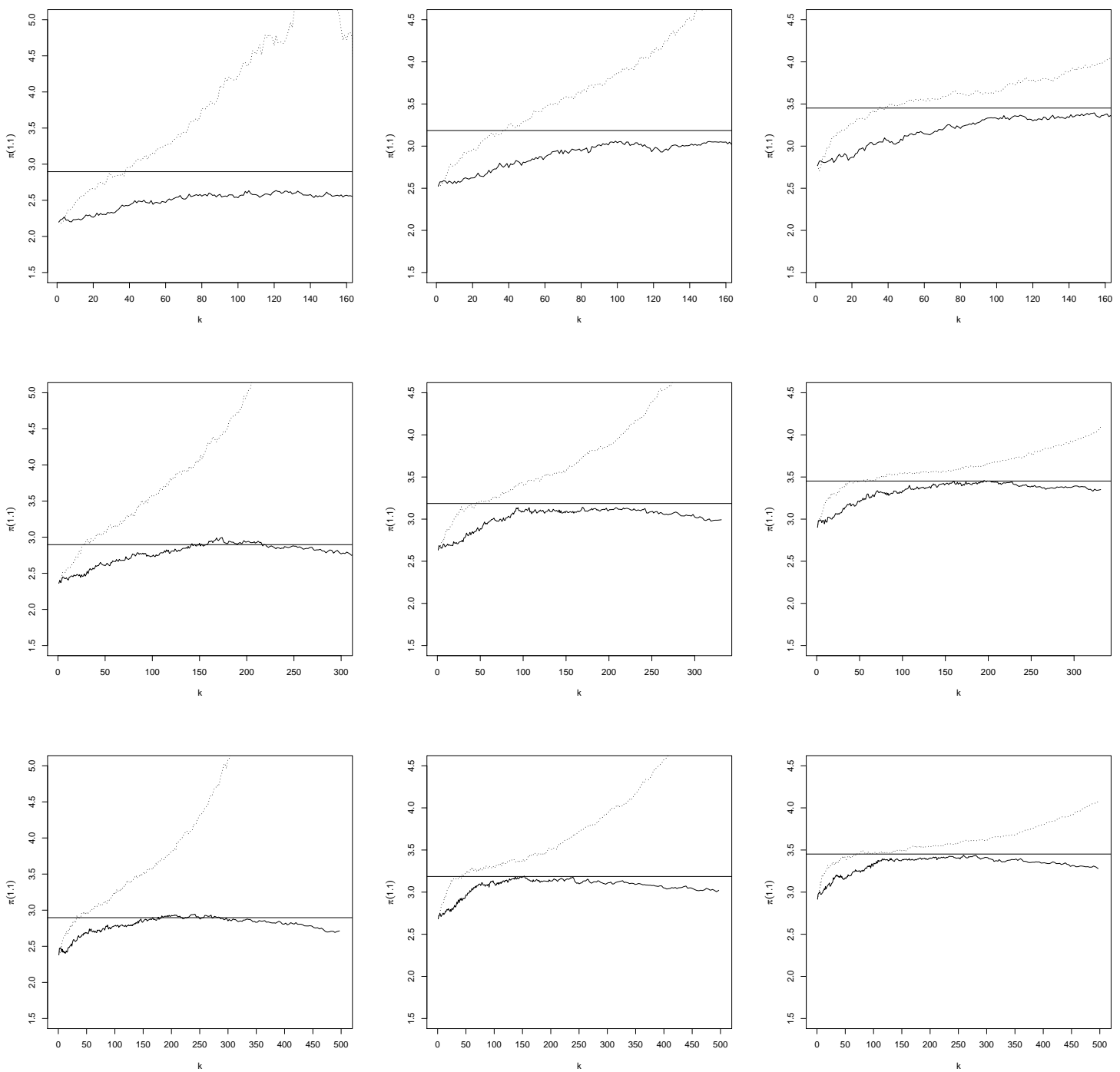

Figure 2: Median of $\widetilde{\pi}_{n, k}^{K}(1.1)$ (dotted line) and $\widehat{\pi}_{n, k}^{\boldsymbol{L S}, \widehat{\boldsymbol{\omega}}}(1.1)$ (full line) as a function of $k$ based on 500 samples of size $n=500$ (top), $n=1000$ (middle) and $n=1500$ (bottom) from a Burr distribution defined as $\bar{F}(x)=\left(1+x^{-\frac{3 \omega}{2}}\right)^{1 / \omega}$. From the left to the right: $\omega=-0.75, \omega=-1$ and $\omega=-1.5$. The horizontal line represents the true value of the premium. 

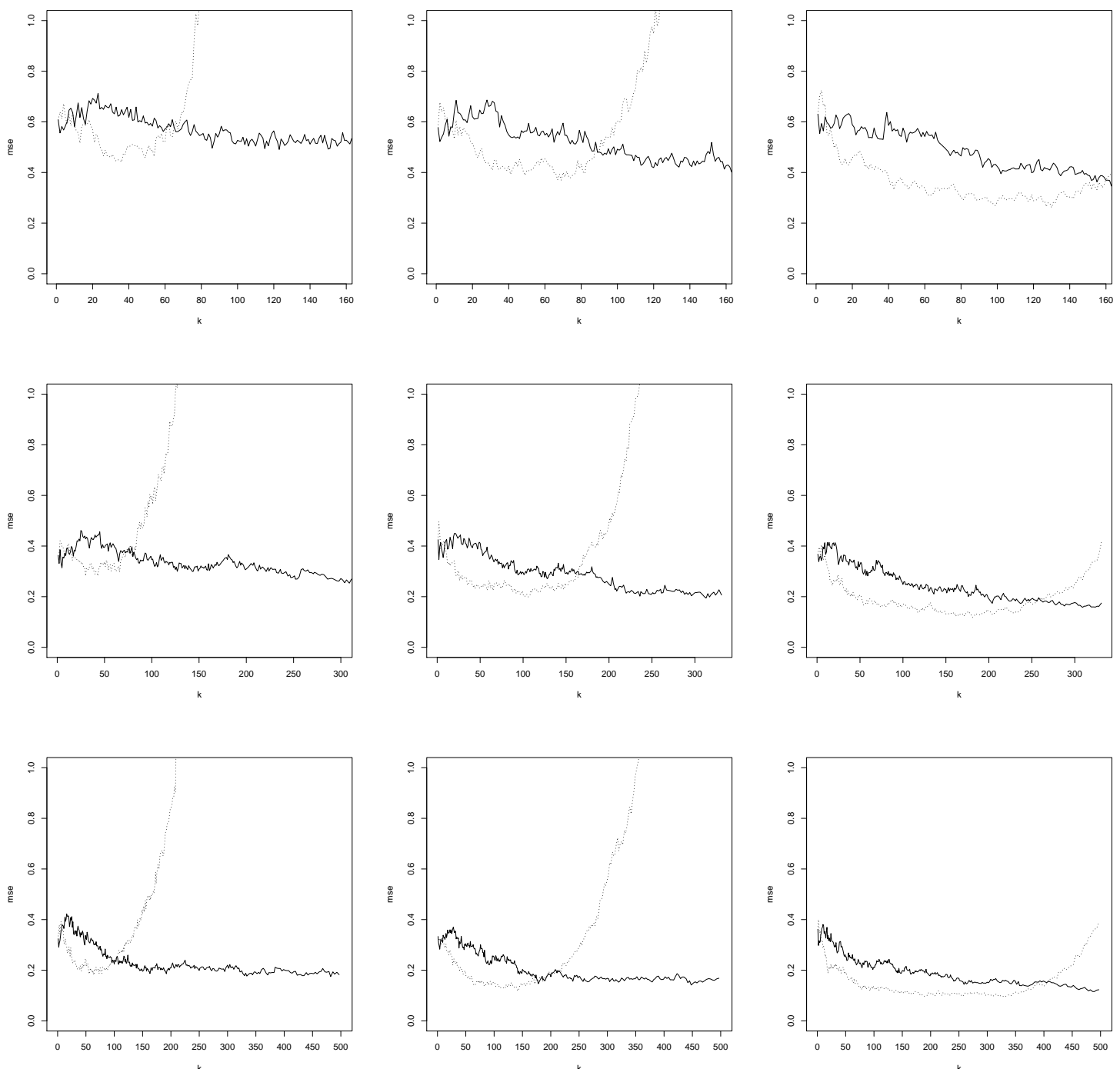

Figure 3: MSE of $\widetilde{\pi}_{n, k}^{K}(1.1)$ (dotted line) and $\widehat{\pi}_{n, k}^{\boldsymbol{L S}, \widehat{\boldsymbol{\omega}}}$ (1.1) (full line) as a function of $k$ based on 500 samples of size $n=500$ (top), $n=1000$ (middle) and $n=1500$ (bottom) from a Burr distribution defined as $\bar{F}(x)=\left(1+x^{-\frac{3 \omega}{2}}\right)^{1 / \omega}$. From the left to the right: $\omega=-0.75, \omega=-1$ and $\omega=-1.5$. 